\title{
What drives high flow events in the Swiss Alps? Recent developments in wavelet spectral analysis and their application to hydrology
}

\author{
B. Schaefli ${ }^{a, *}$, D. Maraun ${ }^{b, 1}$, M. Holschneider ${ }^{c}$ \\ ${ }^{a}$ Institute of Geoecology, University of Potsdam, 14476 Golm, Germany \\ ${ }^{\mathrm{b}}$ Institute of Physics, University of Potsdam, 14469 Potsdam, Germany \\ ${ }^{c}$ Institute of Mathematics, University of Potsdam, 14469 Potsdam, Germany
}

Received 16 January 2007; received in revised form 21 June 2007; accepted 21 June 2007

Available online 3 July 2007

\begin{abstract}
Extreme hydrological events are often triggered by exceptional co-variations of the relevant hydrometeorological processes and in particular by exceptional co-oscillations at various temporal scales. Wavelet and cross wavelet spectral analysis offers promising timescale resolved analysis methods to detect and analyze such exceptional co-oscillations. This paper presents the state-of-the-art methods of wavelet spectral analysis, discusses related subtleties, potential pitfalls and recently developed solutions to overcome them and shows how wavelet spectral analysis, if combined to a rigorous significance test, can lead to reliable new insights into hydrometeorological processes for real-world applications. The presented methods are applied to detect potentially flood triggering situations in a high Alpine catchment for which a recent re-estimation of design floods encountered significant problems simulating the observed high flows. For this case study, wavelet spectral analysis of precipitation, temperature and discharge offers a powerful tool to help detecting potentially flood producing meteorological situations and to distinguish between different types of floods with respect to the prevailing critical hydrometeorological conditions. This opens very new perspectives for the analysis of model performances focusing on the occurrence and non-occurrence of different types of high flow events. Based on the obtained results, the paper summarizes important recommendations for future applications of wavelet spectral analysis in hydrology.
\end{abstract}

(C) 2007 Elsevier Ltd. All rights reserved.

Keywords: Continuous wavelet transform; Wavelet spectrum; Wavelet coherence; Cross wavelet analysis; Time series analysis; Hydrological modelling; Hydrological processes; Catchment hydrology

\section{Contents}

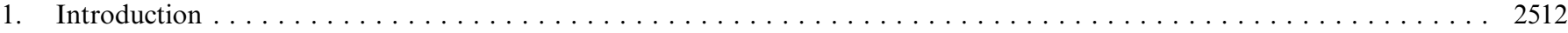

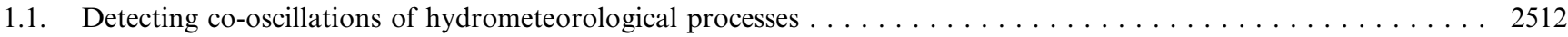

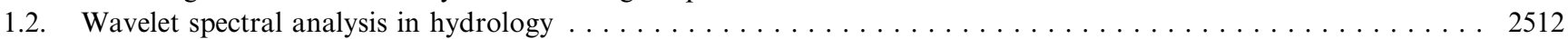

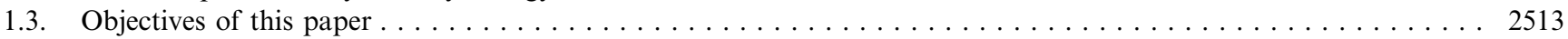

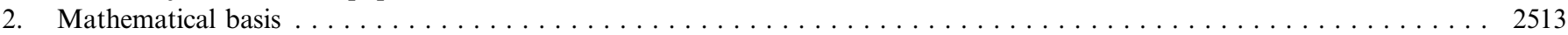

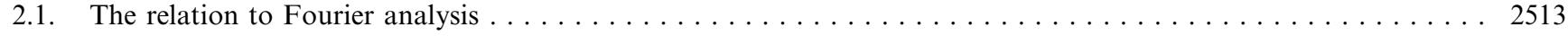

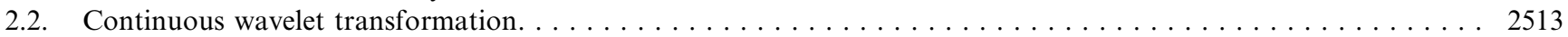

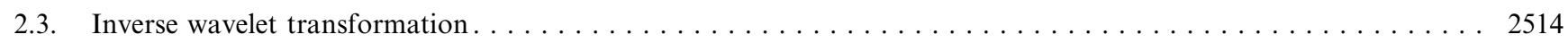

\footnotetext{
* Corresponding author. Present address: Water Resources Section, Delft University of Technology, 2600 GA Delft, The Netherlands. Tel.: +31 15 278 5080 .

E-mail address: bettina.schaefli@a3.epfl.ch (B. Schaefli).

${ }^{1}$ Present address: Climatic Research Unit, University of East Anglia, Norwich NR4 7TJ, UK.
} 


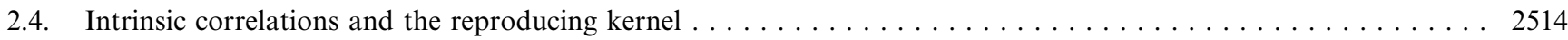

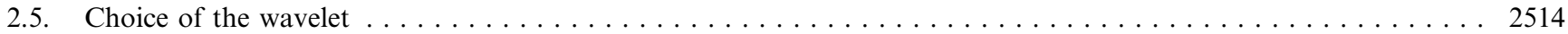

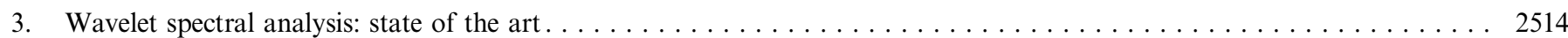

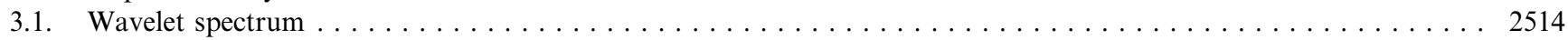

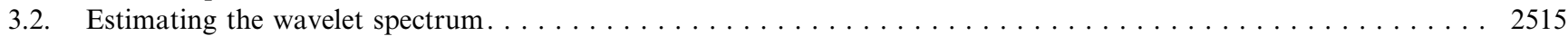

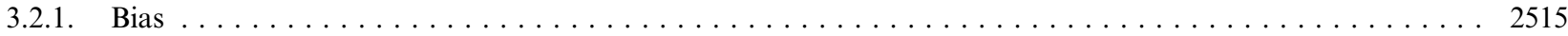

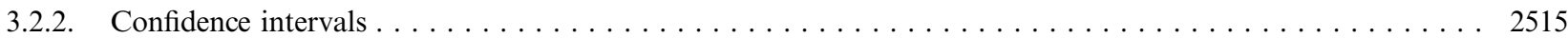

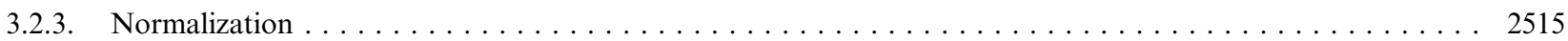

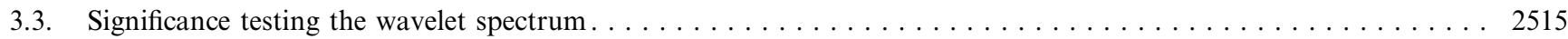

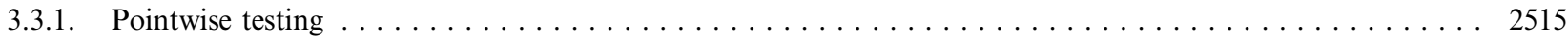

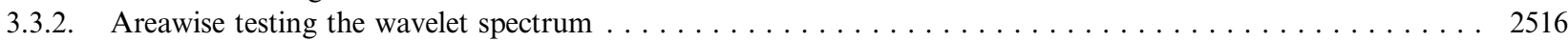

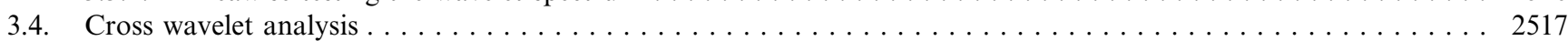

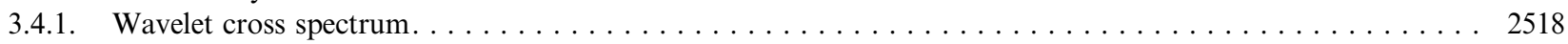

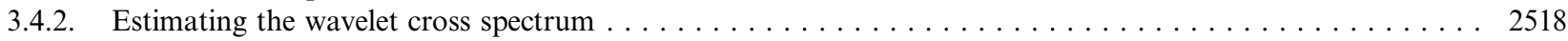

3.4.3. Significance testing the wavelet cross spectrum $\ldots \ldots \ldots \ldots \ldots \ldots \ldots \ldots$

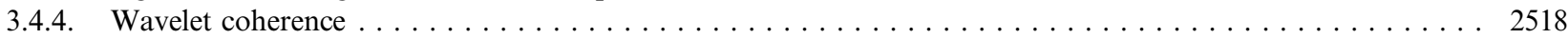

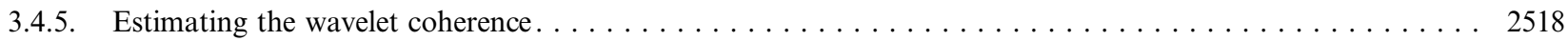

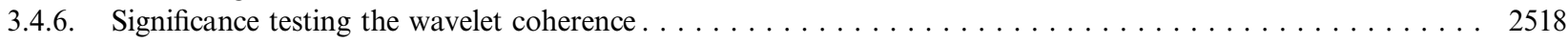

3.4.7. Interpretation of significant wavelet coherence $\ldots \ldots \ldots \ldots \ldots \ldots \ldots \ldots$

4. Case study: detecting flood triggering situations in an Alpine catchment . . . . . . . . . . . . . . . . . 2519

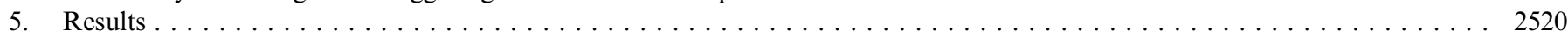

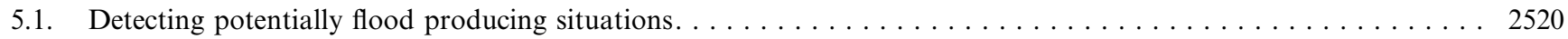

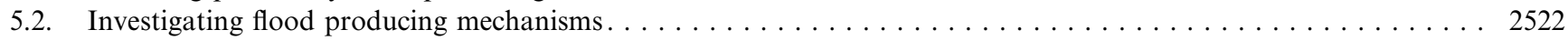

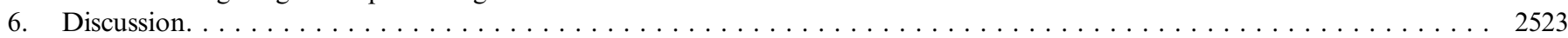

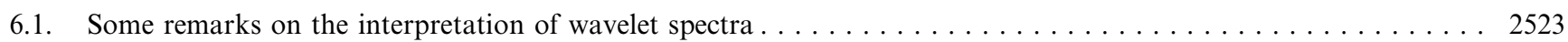

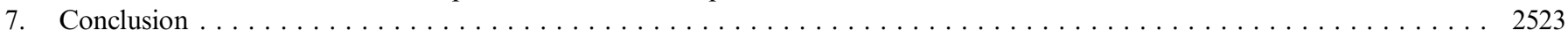

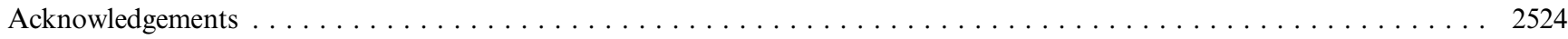

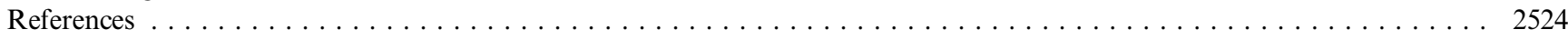

\section{Introduction}

\subsection{Detecting co-oscillations of hydrometeorological processes}

Understanding the dependence between meteorological and hydrological processes is a prerequisite for hydrological modelling, especially for the prediction of extreme hydrological events that are often triggered by exceptional co-variations of the input processes. While general assumptions about the interrelations between driving processes and catchment state or output processes can be derived from our current understanding of hydrological processes, the detection of exceptional situations in the observed time series is not straightforward. Different statistical analysis tools can reveal how the timing and the magnitude of the events are related (e.g. correlation analysis or copulas e.g. [1]. Exceptional situations are, however, often triggered by a co-variation of processes (e.g. precipitation, temperature and soil moisture) at various temporal scales, which can be analyzed by investigating when and at which temporal scales these processes are co-oscillating, i.e. by a timescale (or frequency) resolved analysis.

One method for such a time-scale resolved analysis is wavelet analysis: it decomposes a signal into a superposition of scaled and translated versions of an original (mother) wavelet (a fast-decaying oscillating function). This method has initially been formalized by Grossmann and Morlet [2] and offers a complementary approach to windowed Fourier transformation as proposed by Gabor [3]: a windowed Fourier transformation assumes local stationarity in a time window shifted over the data set, which implies a constant resolution, given by the window size, for all analyzed frequencies. In wavelet analysis, the relative frequency resolution $\Delta f / f$ is constant and the time resolution is adjusted to the analyzed scale (see, e.g. [4,5]).

Wavelet analysis covers a wide range of methods and applications (in addition to the above references, see, e.g. [6], for applications in geosciences, see [7]). In the present paper, we are interested in the co-oscillating properties of observed processes and we, therefore, focus on one particular application that is becoming increasingly popular for hydrological case studies: continuous wavelet spectral analysis based on the continuous wavelet transformation (CWT) (Section 2.2).

\subsection{Wavelet spectral analysis in hydrology}

Early applications of the CWT to hydrology are presented in [8]. The method became increasingly popular after the work published by Torrence and Compo [9], who placed continuous wavelet analysis into the framework of statistical data analysis by formulating significance tests against reasonable background spectra (the Null hypothesis). In river regime characterization, it is used to detect how discharge is related to climate variability indices e.g. [10-12] or to qualitatively analyze how certain features of the meteorological input time series are transferred to 
the hydrological system output e.g. [13,14]. This approach is also applied for the analysis of the hydrological functioning of karstic systems e.g. [15]. Smith et al. [16] applied CWT to answer the question to which degree a catchment smoothes out the spatial variability of rainfall. $\mathrm{Si}$ and Zeleke [17] used CWT to investigate the relationship between hydraulic properties and physical soil properties. In the most recent type of applications, CWT is used to investigate the relationship between measured and simulated hydrographs to assess the performance of hydrological models e.g. [18].

\subsection{Objectives of this paper}

One of the most important difficulties in wavelet spectral analysis concerns the significance testing of wavelet spectra: the hitherto applied (pointwise) significance test detects many false positive peaks, which are often misinterpreted as physical results. We discuss this topic in reasonable detail and present an areawise significance test recently developed by Maraun et al. [19] (see Section 3.3.2). Another typical problem is the cross wavelet analysis of two observed processes that found its application in different areas of geosciences e.g. [9]. However, as this measure is not normalized, its interpretation is not straightforward (see Section 3.4) and wavelet coherence should be used instead (see Section 3.4.4).

Therefore, the objective of this paper is twofold:

- Presenting the state-of-the-art methods of wavelet spectral analysis and highlighting the difficulties and subtleties of estimating and interpreting wavelet spectra.

- Applying these methods to a real-world application to discuss how and why wavelet spectral analysis can give reliable insights into multivariate hydrological processes.

First, in Section 2, we introduce the well known basics of wavelet analysis as well as more subtle relations like the reproducing kernel, which are essential for the further discussion. Readers familiar with these advanced mathematical aspects of wavelet analysis may skip this section. In Section 3, we present the state-of-the-art of continuous wavelet spectral analysis. Part of the discussed methods are classically known in continuous wavelet analysis, but are recalled here because they are not always applied in hydrology (e.g. the use of the wavelet coherence instead of the wavelet cross spectrum e.g. [20]). Some methods have only recently been introduced, namely the areawise significance test [19].

In Section 4, we apply the presented methods to analyze potentially flood producing situations in a catchment in the Swiss Alps for which a recent re-estimation of design floods encountered significant problems simulating the observed high flows. Based on the obtained results, we discuss how continuous wavelet analysis combined to prior knowledge of the relevant physical mechanisms can contribute to the understanding and modelling of hydrological processes (Section 6) and summarize the most important findings and recommendations for future research (Section 7).

\section{Mathematical basis}

In the following, we shortly introduce the mathematics that are the most relevant for the present paper. For a detailed discussion of CWT basics please refer to the comprehensive literature $[4,5,21]$.

\subsection{The relation to Fourier analysis}

The calculation of the Fourier transformation $f(\omega)$ of a time series $s(t)$ reveals information about the frequency content of the underlying process:

$$
\begin{aligned}
& f(\omega)=F\{s(t)\}=\int_{-\infty}^{\infty} \mathrm{d} t s(t) \mathrm{e}^{-\mathrm{i} \omega t}, \\
& s(t)=F^{-1}\{f(\omega)\}=\frac{1}{2 \pi} \int_{-\infty}^{\infty} \mathrm{d} \omega f(\omega) \mathrm{e}^{\mathrm{i} \omega t},
\end{aligned}
$$

where $\omega$ denotes frequency. The Fourier periodogram (based on the discretized version of Eq. (1)) $|f(\omega)|^{2}$ estimates the spectrum of the underlying process at frequency $\omega$. The Fourier spectrum intrinsically assumes stationarity of the analyzed process. Real world processes, however, are often non-stationary, i.e. their statistical correlation properties change over time. Thus a time-independent description of the spectrum is no longer suitable. When extending the concept of the stationary spectrum to time and frequency resolved measures, one faces the problem that a higher time resolution always comes along with a lower frequency resolution and vice versa. This is an immediate consequence of the Heisenberg uncertainty relation. Therefore one needs to find a compromise between the time uncertainty and the frequency uncertainty in the description of the process. One possible compromise is provided by wavelet analysis: here, the time resolution is chosen proportional to the scale (i.e. periodicity) to be analyzed. Another compromise would be time-windowed Fourier analysis where the time resolution is constant for all analyzed frequencies. Many more approaches are possible, and much of what we expose in the following can easily be carried over to other time-frequency techniques as well.

\subsection{Continuous wavelet transformation}

The continuous wavelet transform (CWT) of a time series $s(t), W_{g}(b, a)$, at time $b$ and scale $a$ with respect to the chosen wavelet $g(t)$ is given as

$W_{g}(b, a)=W T_{g}\{s(t)\}=\int \mathrm{d} t \frac{1}{\sqrt{a}} \bar{g}\left(\frac{t-b}{a}\right) s(t)$,

where $\left(^{\cdot}\right)$ denotes complex conjugate. Here, we choose the $L^{2}$-normalization $1 / a^{\frac{1}{2}}$ which will appear to be a natural choice (see Section 3). 
The exponential function in Eq. (1) has been replaced by a localized wavelet $g(t)$ which is translated by the time $b$ and stretched according to the scale $a$. Accordingly, wavelet analysis offers a time as well as frequency resolved analysis.

\subsection{Inverse wavelet transformation}

An inverse wavelet transformation of a function $r(b, a)$ from the wavelet domain $\mathbb{U}$ to the time domain can be defined as follows:

$$
\begin{aligned}
M_{h}(t) & =I W T_{h}\{r(b, a)\} \\
& =\int_{\mapsto} \frac{\mathrm{d} b \mathrm{~d} a}{a^{2}} r(b, a) \frac{1}{\sqrt{a}} h\left(\frac{t-b}{a}\right) .
\end{aligned}
$$

Here, $h(t)$ is called a reconstruction wavelet that, under some very weak assumptions, can be derived from $g(t)$. If $g(t)$ is scaled suitably, then $g(t)$ and $h(t)$ may even be chosen to be identical (for details see [5]). In case that $r(b, a)=W T_{g}\{s(t)\}$, then $I W T_{h}\{r(b, a)\}=s(t)$ reconstructs the original signal ${ }^{2}$.

\subsection{Intrinsic correlations and the reproducing kernel}

The CWT transforms a signal from the one dimensional time domain to the two dimensional time-scale domain. It is therefore not surprising that it produces redundancies that are reflected in intrinsic correlations. This is mostly visible in the wavelet transform of a signal which is completely decorrelated. Indeed, for the wavelet transformation of Gaussian white noise $\eta(t)$, a simple relation between the wavelet used for the analysis and the correlations exists: the intrinsic correlations between the wavelet coefficients at $(b, a)$ and $\left(b^{\prime}, a^{\prime}\right)$ are given (up to some factor) by the so-called reproducing kernel $K_{g, g}\left(\left(b-b^{\prime}\right) / a^{\prime}, a / a^{\prime}\right)$ moved to the time $b^{\prime}$ and stretched to the scale $a^{\prime}[5,21]$ :

$C\left(b, a ; b^{\prime}, a^{\prime}\right) \sim K_{g, g}\left(\frac{b-b^{\prime}}{a^{\prime}}, \frac{a}{a^{\prime}}\right)$

The reproducing kernel itself is given as the wavelet transformation of the reconstruction wavelet, $K_{g, h}\left(\left(b-b^{\prime}\right) / a^{\prime}\right.$, $\left.a / a^{\prime}\right)=W T_{g}\left\{h\left(\left(b-b^{\prime}\right) / a^{\prime}\right)\right\}$. The effect of intrinsic correlations appears in every time-frequency analysis and represents a time-scale uncertainty.

\subsection{Choice of the wavelet}

In principle, there is a wide range of wavelets available. We roughly distinguish two types of wavelets: real valued wavelets and progressive wavelets (i.e. wavelets which have

\footnotetext{
${ }^{2}$ In case of a progressive wavelet (for a definition see Section 2.5) and $r(b, a)$ being the wavelet transformation of a real valued signal $s(t)$ (which is the most common situation), the above formula will rather recover the analytic signal associated with $s(t)$. Its real part however is again $s(t) / 2$, $2 \operatorname{Re} M_{h}(t)=s(t)$.
}

only positive frequencies). The latter ones are necessarily complex valued. This is why those wavelets are sometimes (erroneously) simply called complex wavelets. For details see (see, e.g. [4,5]). However, the special requirements of a time and scale resolved spectral analysis (which is the focus of this paper) restrict the choice in the following way:

- As one is interested in amplitudes of frequency content it is preferable to use progressive, linear phase wavelets (i.e. wavelets with a real valued Fourier transform). These wavelets clearly separate amplitude and phase information. For instance the wavelet transform of a pure sinusoidal signal has a time independent distribution of energy. Using a real valued wavelet, the amplitude and the phase information would mix, producing oscillating energy in the wavelet plane. Using a progressive wavelet, the amplitude is constant and the phase picture of the wavelet plane may be used to estimate the phase of the oscillation.

- To obtain a reasonable localization of the analysis in both time and scale, a wavelet is required that exhibits a good localization in the time domain as well as in the frequency domain (in other words: the reproducing kernel should not be too extended neither in the time nor in the scale direction). Thus, for instance the Haar wavelet (in addition to the fact that the Haar wavelet is real) is not suitable for non-stationary wavelet spectral analysis. Its poor regularity (it has jumps) implies a very slow decay of its spectrum with many side peaks. In a spectral analysis even of a pure frequency, this would lead to corresponding side peaks of energy in the wavelet plane.

- The trade-off between time and scale resolutions has to be adapted to the analyzed process. A Morlet wavelet with $w_{0}=2 \pi$ has been shown to work well for different hydrological applications where the main objective is to analyze observed time series e.g. $[11,17,14]$. Since the Morlet wavelet is strictly speaking no wavelet (its mean does not exactly vanish), it produces artifacts for small values of $w_{0}$. However, using only values of, say, $w_{0} \geqslant \pi$ one can adjust the time-frequency resolution for the individual analysis. Lane [18] uses a complex Mexican hat wavelet (i.e. the analytic function of the second derivative of a Gaussian) to have a very good time resolution for the analysis of individual flood peaks.

\section{Wavelet spectral analysis: state of the art}

\subsection{Wavelet spectrum}

A stationary Gaussian process is fully described by its Fourier power spectrum $S(\omega)=|f(\omega)|^{2}$. Realizations of such a process can be constructed by filtering Gaussian white noise in the Fourier domain, i.e. $s(t)=F^{-1}\{f(\omega) \cdot F\{\eta(t)\}\}$, where $\eta(t)$ is a realization of Gaussian white noise. In a 
similar way, we introduce a class of processes by filtering in a non-stationary way the wavelet transform of white noise and transforming back. More precisely, for a fixed analysisreconstruction pair $(g, h)$, we choose a wavelet filter function $m(b, a)$. The spectrum of the process is then defined as

$S(\omega)=|m(b, a)|^{2}$.

Here $m(b, a)$ is a complex function in time $b$ and scale $a$ following a certain asymptotic behavior (for details see [19]) to ensure the well definiteness of the process. Within this framework, white noise is defined as $m(b, a) \equiv$ const., i.e. independent of time and scale. Realizations of a nonstationary Gaussian process following this spectrum are generated by

$s(t)=I W T_{h}\left\{m(b, a) \cdot W T_{g}\{\eta(\tau)\}\right\}$,

with $\eta(\tau)$ being Gaussian white noise. These realizations depend on the wavelets $g(t)$ and $h(t)$, thus for a complete definition of the process, one needs the spectrum as well as the wavelets chosen for the filtering. For a discussion of rather subtle implications, which are beyond the scope of this paper, refer to [19].

\subsection{Estimating the wavelet spectrum}

Given a time series $s(t)$, the underlying wavelet spectrum can be estimated by the wavelet sample spectrum

$\widehat{S}_{g}(b, a)=A\left(\left|W_{k} s(t)\right|^{2}\right)$,

where $k(t)$ denotes the wavelet chosen for the analysis. $A(\cdot)$ denotes an averaging kernel to reduce the variance of the estimate. This kernel needs to be adjusted to the time and frequency resolution on every scale, i.e. to the reproducing kernel, see Section 2.4:

- For averaging over a range of scales, the width of the kernel should be proportional to scale, i.e. for the common logarithmic scale axis, the width would be constant.

- For averaging over a certain time region, the width of the kernel has to be chosen proportional to the scale under consideration, such that always the same number of oscillations fit into the kernel.

For details about the averaging please refer to [19].

\subsubsection{Bias}

This estimator is not unbiased. The averaging produces an averaging bias, and an additional bias results from the intrinsic correlations (Eq. (4)), i.e. from the time-frequency uncertainty relation inherent to any time-frequency analysis [19]. Due to this intrinsic bias, the wavelet sample spectrum is not a consistent estimator of the true wavelet spectrum for finite scales. This bias might initially appear of rather theoretical interest, but becomes more obvious when considering a time series analyzed with two different wavelets: for finite scales, the resulting wavelet sample spec- trum will be different for both analyses and both will be biased against the true wavelet spectrum.

\subsubsection{Confidence intervals}

The variability of the estimator can be estimated by generating a bootstrap ensemble using Eq. (6) (see [19]). When using white noise for the generation, this implicitly assumes Gaussianity of the process. From the bootstrap ensemble, one can easily derive an arbitrary confidence region, e.g. $95 \%$ confidence intervals, for every point in the wavelet domain.

If the marginal distribution of the data deviates strongly from Gaussianity, these confidence intervals might be misleading. For a general introduction to bootstrapping, please refer to e.g. [22].

\subsubsection{Normalization}

Except for the bias discussed above, the chosen $L^{2}$-norm in Eq. (2) directly relates the estimator $A\left(\left|W_{g} s(t)\right|^{2}\right)$ to the true wavelet spectrum $|m(b, a)|^{2}$. For instance, in the case of white noise, $m(b, a) \equiv$ const., the expectation value of the estimator is time and scale independent, $E\left(A\left(\left|W_{g} s(t)\right|^{2}\right)\right)$ $\equiv$ const. Using other normalization factors, the estimated wavelet spectrum shows a different scale dependency (e.g. a decay for white noise for long scales) and needs to be renormalized to be comparable with the true spectrum. In this sense, the $L^{2}$ normalization is the natural choice for continuous wavelet spectral analysis. However, this normalization overemphasizes power on high scales that might easily be misinterpreted without a significance test. For a discussion of pitfalls related to the interpretation of the $L^{2}$-normalization and other normalizations, please refer to [20].

\subsection{Significance testing the wavelet spectrum}

The wavelet sample spectrum of an observed time series is merely an estimate of the true wavelet spectrum and is thus subject to variance and bias. Accordingly, the quality of the estimate needs to be assessed in statistical terms. This can either be done in terms of confidence intervals, see Section 3.2.2 or by testing the estimated spectrum against a Null hypothesis for the true spectrum (e.g. an AR [1] process that according to Hasselmann [23] is suitable as Null hypothesis for many climatological applications, see also Section 4).

\subsubsection{Pointwise testing}

Torrence and Compo [9] established a pointwise significance test for the wavelet spectrum to judge whether a measured sample spectrum deviates significantly from a trivial background process fitted to the data. A slightly modified version $[20,19]$ is performed as follows:

- Choose a significance level $1-\alpha$.

- Choose a reasonable model as Null hypothesis $H_{0}$ and fit it to the data. 

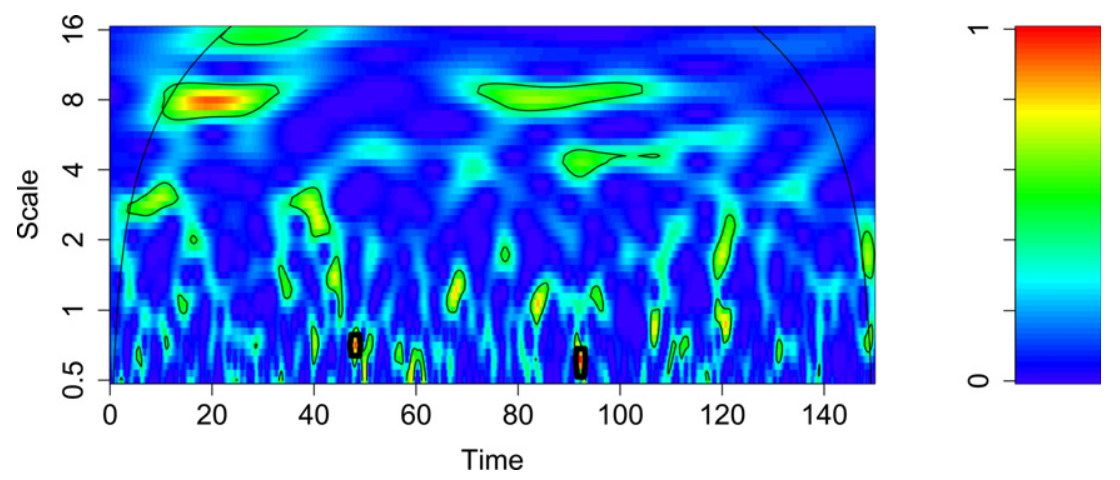

Fig. 1. Wavelet periodogram of a Gaussian white noise realization with the Morlet wavelet, $\omega_{0}=2 \pi$. Thin lines: pointwise test on $95 \%$ level, thick lines: additional areawise test on $90 \%$ level.

- Estimate the $(1-\alpha)$-quantile $S_{\text {crit }}$ (i.e. the critical value) of the corresponding background spectrum by Monte Carlo simulations. Depending on the chosen background model and the chosen normalization of the spectral estimator, the critical value in general depends on scale.

- Check for every point in the wavelet domain, whether the estimated spectrum exceeds the corresponding critical value. The set of all pointwise significant wavelet spectral coefficients is given as

$$
P_{\mathrm{pw}}=\left\{(b, a) \mid \widehat{S}_{g}(b, a)>S_{\mathrm{crit}}\right\} \text {. }
$$

We call this test pointwise as it tests every point in the wavelet domain separately without accounting for any spatial structure. Hitherto, only this test has been applied for statistical evaluations. However, pointwise testing in the time-frequency domain has a severe drawback that almost always leads to misleading false positive results: given a significance level $1-\alpha$, a repetition of the test for $N$ wavelet spectral coefficients leads to, on an average, $\alpha N$ false positive results. This effect is referred to as multiple testing and appears in every case where a significance test is repeated on different samples. For any time-frequency analysis, one faces a second problem: according to Section 2.4, neighboring wavelet coefficients are correlated, i.e. false positive values are unlikely to occur alone, but always as a contiguous patch. Thus, the wavelet plot of a time series without any interesting signal will by construction be covered by many false positive patches, making up about $\alpha$ percent of the wavelet plot area ${ }^{3}$. These effects mark an important problem: without additional information, it is not clear if patches detected in a pointwise test reflect real physical behavior or simply represent artifacts emerging from multiple testing effects and the intrinsic correlations of the wavelet transformation.

\footnotetext{
${ }^{3}$ Here it is important to note, that the area has to be calculated relatively to a scale dependent measure, in other words: a ten year long signal on the one year scale is effectively two times longer than a ten year signal on the two year scale.
}

Fig. 1 illustrates this problem: the plot shows the wavelet periodogram (using a Morlet wavelet with $\omega_{0}=2 \pi$ ) of a Gaussian white noise realization. The thin lines enclose regions of pointwise significance on the $95 \%$ level. However, as the process itself is fully captured by the red noise Null hypothesis, all the detected deviations are false positive results, i.e. artifacts (the thick lines will be discussed in the following section).

\subsubsection{Areawise testing the wavelet spectrum}

The previous discussion illustrates that for a reliable analysis it is necessary to account for the size and spatial structure of the detected patches. Accordingly, Maraun et al. [19] developed an areawise significance test that overcomes the problems discussed in the previous section. The main idea is the following: if the intrinsic correlations are given by the reproducing kernel (Section 2.4), then also the typical patch area for random fluctuations is given by the reproducing kernel. Patches small compared to the reproducing kernel are in principle indistinguishable from noise. This idea leads to the following design of an areawise significance test.

Following the dilation of the reproducing kernel Eq. (4), the typical width in time and scale direction of false positive patches should grow linearly with scale. Thus, one defines critical areas as a projection of the reproducing kernel onto the wavelet domain:

$P_{\text {crit }}(b, a)=\left\{\left(b^{\prime}, a^{\prime}\right) \mid\left(K\left(b, a ; b^{\prime}, a^{\prime}\right)>K_{\text {crit }}\right\}\right.$.

This area represents the effective size of the reproducing kernel given a certain significance level, i.e. the threshold $K_{\text {crit }}$ is determined by the significance level of the areawise test ${ }^{4}$. The latter is defined as the ratio between the area of patches passing the areawise test and the area of patches passing the pointwise test (this area is weighted corresponding to the scale, see previous section). Loosely speak-

\footnotetext{
${ }^{4}$ The determination of the threshold is rather non-trivial: one chooses an initial value for $K_{\text {crit }}$ and calculates the corresponding significance level by a large set of Monte Carlo simulations. Then the value for $K_{\text {crit }}$ is numerically optimized in an iterative procedure to obtain the desired significance level, see [19].
} 
ing, the areawise test on the $10 \%$-level sorts out $90 \%$ of the false positive patches.

Then, given the set of all pointwise significant patches $P_{\mathrm{pw}}$, the subset of additionally areawise significant wavelet patches is given as the union of all critical areas that completely lie inside the patches of pointwise significant values:

$P_{\mathrm{aw}}=\bigcup_{P_{\mathrm{crit}}(b, a) \subset P_{\mathrm{pw}}} P_{\text {crit }}(b, a)$.

In other words: patches thinner or even completely smaller than the critical area are indistinguishable from background noise and consequently rejected by the areawise test. Note that the critical area is stretched according to the analyzed scale (see Eq. (9)), i.e. a patch needs to be larger to be significant on high scales.

To summarize, the actual areawise test is performed as follows:

- Perform the pointwise test according to Section 3.3.1 on the $1-\alpha$ level.

- Choose a significance level for Eq. (4), choose a significance level $1-\alpha_{\text {aw }}$ for the areawise test and the corresponding critical area $P_{\text {crit }}(b, a)$ of the reproducing kernel.

- Slide the critical area $P_{\text {crit }}(b, a)$ (for every scale the corresponding dilated version) over the wavelet matrix. A point inside a patch is defined as areawise significant, if any critical area containing this point totally lies within the patch.

This test has been shown to drastically increase the reliability of a wavelet spectral analysis by rejecting most false positive results. For details, refer to [19].

3.3.2.1. Interpreting areawise significant patches. The areawise test has to be interpreted in the following way: a process that agrees with the Null hypothesis of the pointwise test produces typical patches that are with a probability of about $1-\alpha_{\mathrm{aw}}$ smaller than the critical area. An areawise significant patch can therefore occur in three cases: the first (and trivial) one is a false positive patch which occurs in about $\alpha_{\text {aw }} \%$ of the cases. The two more interesting cases are the following: either the correlation structure of the analyzed process locally deviates from Gaussian red noise, or its marginal distribution is locally strongly nonGaussian.

In the first of the interesting cases, the interpretation is very intuitive: for the Morlet wavelet with $\omega_{0}=2 \pi$, the width of the critical area on a scale $a$ is roughly three times $a$. In other words: one needs to observe at least three cycles of an oscillation to distinguish it from red background noise. Shorter events are in principle invisible, depending on the chosen time-frequency resolution: a high time resolution might enable to detect more localized events at the cost of a low frequency resolution.

The second case addresses extreme events: only extreme events that highly exceed the noise level (which is assumed to be Gaussian) might be identified. These events appear in the wavelet plot as very time-localized patches that reach from the lowest scale to considerably high scales. Such a patch remains areawise significant even though the underlying event is much shorter in time than the reproducing kernel. This rather non intuitive case can be explained as follows: the single high value is convolved with the wavelet on all scales. For small scales, the resemblance is very high, leading to high values of the wavelet transform. Due to the intrinsic correlations, the single event is smeared out to a certain time interval that is long compared to the reproducing kernel. The resulting areawise peak can be identified very easily, as it is very confined in the time direction, usually starts at the lowest analyzed scale and reaches over a wide range of scales. Accordingly, this case does not reflect any correlation structure, but an extreme event visible in the wavelet spectrum due to non-Gaussianity of the data, e.g. a strong asymmetry or a heavy tailed distribution. If only the correlation structure is of interest, a solution to reduce the effect of extreme events is to transform the marginal distribution to follow approximately a Gaussian marginal distribution. This, however, might also affect the correlation structure itself, so any transformation of the marginal distribution should be done carefully, comparing the results of different approaches. (An alternative to transforming the data to a Gaussian marginal distribution could be to model a non-Gaussian Null-hypothesis. This, however, is beyond the scope of this paper.)

The functioning of the areawise test can be illustrated by Fig. 1: most of the pointwise significant patches are small compared to the critical area. For example the two large patches on the 8 years scale are both rejected, the first one at around $t=20$ because it is too short (roughly $21 / 2$ cycles), the second one because it is too slim. Only two patches are attributed "areawise significant". However, these are not detected, because they have got any physical meaning, but because also the areawise test is subject to (a reduced effect of) multiple testing: on the $90 \%$ level, from the $20-30$ patches around 2-3 should by chance be so large that they are false positively detected.

To summarize, the areawise test helps to reject most false positive results. Due to its high specificity, an areawise deviation almost always (depending on the significance level of the areawise test) denotes a true deviation from the Null hypothesis. Hence, this test has the ability to greatly enhance the reliability of wavelet spectral analysis, as many tempting and misleading results are rejected. However, also the areawise test should not be applied blindly; the areawise test produces also false positive results and in certain cases it could be too specific (see Section 6.1).

\subsection{Cross wavelet analysis}

Compared to the wavelet spectral analysis, the inference of covarying power is even more complicated. This is mainly due to two reasons: first of all, even for a zero true wavelet cross spectrum, the estimated wavelet cross 
spectrum will always be non-zero. Thus, peaks in the wavelet cross spectrum are often falsely interpreted. Secondly, even if a peak in the wavelet cross spectrum or wavelet coherence really signifies common oscillations, this does not necessarily imply a relationship between the two processes. This is due to the local character of wavelet analysis: even two independent processes, as long as they oscillate with not too different frequencies, will randomly oscillate together for finite time intervals.

\subsubsection{Wavelet cross spectrum}

The wavelet cross spectrum

$$
C S(b, a)=m_{1 c}(b, a) \bar{m}_{2 c}(b, a)
$$

defines two covarying non-stationary Gaussian processes, where $m_{1 c}$ and $m_{2 c}$ describe the covarying fractions of the overall spectra given by $m_{1}(b, a)=m_{1 c}(b, a)+m_{1 i}(b, a)$ and $m_{2}(b, a)=m_{2 c}(b, a)+m_{2 i}(b, a)$. Here, $m_{1 i}$ and $m_{2_{i}}$ are independent contributions to the variance. Realizations can be calculated according to Eq. (6) with identical noise for the covarying parts and independent noise for the independently varying parts.

In general, this is a complex function that may be decomposed into amplitude and phase:

$$
C S(b, a)=|C S(b, a)| \exp (\mathrm{i} \arg (C S(b, a)))
$$

For independent processes, the true wavelet cross spectrum is zero.

\subsubsection{Estimating the wavelet cross spectrum}

Given realizations $s_{1}(t)$ and $s_{2}(t)$ of two processes, the wavelet cross spectrum of the underlying process can be estimated by the wavelet sample cross spectrum

$\widehat{C S}_{g}(b, a)=A\left(W_{g} s_{1}(t) \bar{W}_{g} s_{2}(t)\right)$,

or decomposed into amplitude and phase,

$\widehat{C S}_{g}(b, a)=\left|\widehat{C S}_{g}(b, a)\right| \exp \left(i \arg \left(\widehat{C S}_{g}(b, a)\right)\right)$.

Also here, confidence intervals can be estimated by generating a bootstrap ensemble.

\subsubsection{Significance testing the wavelet cross spectrum}

Torrence and Compo [9] and Huang et al. [24] suggested a test for the wavelet cross spectrum against two red noise processes fitted to the two data sets to be analyzed. However, Maraun and Kurths [20] showed that this test is not specific and by a misconception has to lead to wrong results. In fact, such as for the stationary Fourier cross spectrum, no significance test for the wavelet cross spectrum exists. Assume two processes exhibiting independent power at overlapping time and scale intervals. This power does not covary, i.e. information about one of the processes is not capable of predicting the other one. Hence, the real wavelet cross spectrum is zero. By contrast, the estimated wavelet cross spectrum always differs from zero, even if the processes are completely independent (this is a finite size effect; the sample covariance of two independent white noise realizations of finite noise will also be different from zero). As the wavelet sample cross spectrum is not a normalized measure, significant peaks will occur not only in case of covarying power, but also if one (or both) of the single spectra exhibits strong power. Maraun and Kurths [20] investigated the wavelet cross spectrum between the NINO3-index and the NAO-index and showed that peaks do not occur because of a (presumed) El Niño influence on the NAO, but only because of the strength of the El Niño itself. To overcome this problem, one normalizes the cross spectrum and tests against zero coherence.

\subsubsection{Wavelet coherence}

The normalized squared modulus of the wavelet cross spectrum yields the squared wavelet coherence,

$$
\begin{aligned}
\operatorname{COH}^{2}(b, a) & =\frac{|C S(b, a)|^{2}}{S_{1}(b, a) S_{2}(b, a)} \\
& =\frac{\left|m_{1 c}(b, a) \bar{m}_{2 c}(b, a)\right|^{2}}{\left|m_{1}(b, a)\right|^{2}\left|m_{2}(b, a)\right|^{2}} .
\end{aligned}
$$

This measure exhibits values between 0 and 1 , signifying a vanishing and a perfect linear relationship, respectively.

\subsubsection{Estimating the wavelet coherence}

Given realizations $s_{1}(t)$ and $s_{2}(t)$ of two processes, the squared wavelet coherence of the underlying process can be estimated by the squared wavelet sample coherence as

$$
\widehat{C O H}_{g}^{2}(b, a)=\frac{\left|\widehat{C S}_{g}(b, a)\right|^{2}}{\widehat{S}_{g, 1}(b, a) \widehat{S}_{g, 2}(b, a)} .
$$

For the sample coherence, averaging is essential. Already in Fourier coherence analysis, considering only one frequency bin, the statistical concept of coherence breaks down: two pure harmonic oscillations of identical frequency are always perfectly coherent. For wavelet coherence, the problem is even more obvious: investigating power in a single point in time and scale prevents any information about the oscillation and thus covariance. Consequently, numerator and denominator become equal and one obtains a trivial value of one for any two processes.

\subsubsection{Significance testing the wavelet coherence}

Also for wavelet coherence, one should always apply the pointwise test in combination with the areawise significance test.

The pointwise test for wavelet coherence differs from that for the wavelet spectrum in two ways: firstly, as the coherence is normalized to the single wavelet spectra, the critical value becomes independent of the scale as long as the smoothing is done properly according to Section 3.2, i.e. when the geometry of the reproducing kernel is accounted for [20]. Secondly, because of the normalization the coherence should also asymptotically become process independent. As wavelet analysis is localized, one is in gen- 
eral not in the asymptotic regime. However, Maraun et al. [19] showed that the process dependency is weak and might be ignored in a good approximation.

The procedure for the areawise test is the same as for the wavelet spectrum, only the critical patch-size $P_{\text {crit }}(b, a)$ has to be re-estimated. Areawise significant patches denote significant common oscillations of two processes. Here, common means that two processes exhibit a rather stable phase relation on a certain scale for a certain time interval.

\subsubsection{Interpretation of significant wavelet coherence}

The interpretation of the areawise test for coherence is similar as for the spectrum. Besides a true physical coupling of the processes and a mere false positive result, two other cases exist.

3.4.7.1. Coincidence of extreme events. Similar to the wavelet spectrum that can exhibit areawise significant patches for extreme events, areawise significant patches might occur in the wavelet coherence when two extreme events coincide. Such a patch does then not reflect a co-oscillation or coherence of the two processes on a certain scale but rather a coincidence of extremes of both processes. As mentioned previously, if one is not interested in these coincidences, the marginal distributions of the time series could be transformed to obtain a more Gaussian distribution (see also Section 5). Also here, any transformation should be applied carefully, as it might also destroy interesting coherence.

3.4.7.2. Random co-oscillation. An areawise significant result does not necessarily imply coherence in a strict sense. Processes oscillating on similar frequencies trivially exhibit patches indicating an intermittently similar phase evolution. The lengths of the patches are given by the decorrelation times of the single processes and the similarity of the concerned frequencies.

In many geophysical applications, this problem can be solved considering system dynamics and not only statistics. If the results of a wavelet spectral analysis suggest two physically possible alternative explanations or if the knowledge about the underlying dynamics is insufficient, further statistical analysis is required to investigate if the detected coherence is due to a randomly common oscillation or if it really signifies a coupling between the two processes: one has to test if the time interval of the common oscillations is significantly long compared to typical randomly common oscillations of independent processes. The length distribution under the Null hypothesis (i.e. of randomly common oscillations) can be estimated by a bootstrap approach. For an overview, please refer to [19].

\section{Case study: detecting flood triggering situations in an Alpine catchment}

The presented methods are used to investigate the flood producing mechanisms in a catchment located in the Swiss
Alps, the Vispa River at Visp (7.87 E, $46.30 \mathrm{~N}$, catchment size $778 \mathrm{~km}^{2}$ ). This river lies within the catchment of the Swiss part of the Rhone River, for which flood prevention is currently the subject of intense research e.g. [25] and for which the design floods have recently been re-estimated [26]. This estimation was based on a continuous simulation framework including a hydrological model [27] coupled to a stochastic weather generator [28] that generates temperature and precipitation fields based on general circulation indices e.g. [29] and on an analogue sorting method e.g. [30]).

The Vispa river, as well as the entire Swiss Rhone catchment, has experienced four major flood events during the last 20 years e.g. [31], at the end of August 1987, at the end of September 1993 and 1994 and mid-October 2000 (Fig. 2). These floods where presumably mainly induced by exceptional rainfall events; however, in Alpine catchments such as the Vispa catchment (altitude ranging from around $650-4500 \mathrm{~m}$ a.s.1., mean altitude around $2600 \mathrm{~m}$ a.s.1., $30 \%$ of the catchment covered by glacier), the occurrence of floods is strongly influenced by the joint action of rainfall and temperature that determines whether precipitation falls as rain or accumulates as snow. Another important factor is the catchment storage capacity before a heavy rainfall event starts: if the soils or the snow cover are almost saturated, a rainfall event will lead to much higher discharges than in situations where the storage potential is high. As a result, similar precipitation events can lead to completely different discharge situations. The flood of 2000 was for example much less severe in the Vispa catchment than in the rest of the Rhone catchment [32].

The simulation of extreme high flow events in Alpine catchments is particularly difficult due to the high spatial variability of the main system inputs, i.e. of the precipitation and of the temperature. For the Vispa catchment, the application of a conceptual hydrological model performing satisfactorily in neighboring catchments was not able to reproduce all four observed high flow events [26]. Furthermore, the weather generator designed to reproduce the observed statistics of the precipitation and the temperature fields [28] induced simulated extreme discharge events during spring, a period during which exceptional high flows never occurred in this area. Based on these observations, the question arises which particular hydrometeorological situations induced the four observed floods in this particular catchment and whether other similar situations occurred during the observed period without, however, generating high discharges. Investigating the hydrological model performance for these situations would enable a more meaningful assessment of the performance of the hydrological model than just considering the four observed extreme events.

In the following, we will show whether and how the dominant driving process for the occurrence of floods can be detected through wavelet spectral analysis of the observed discharge and the precipitation and temperature observed at a meteorological station located within the 

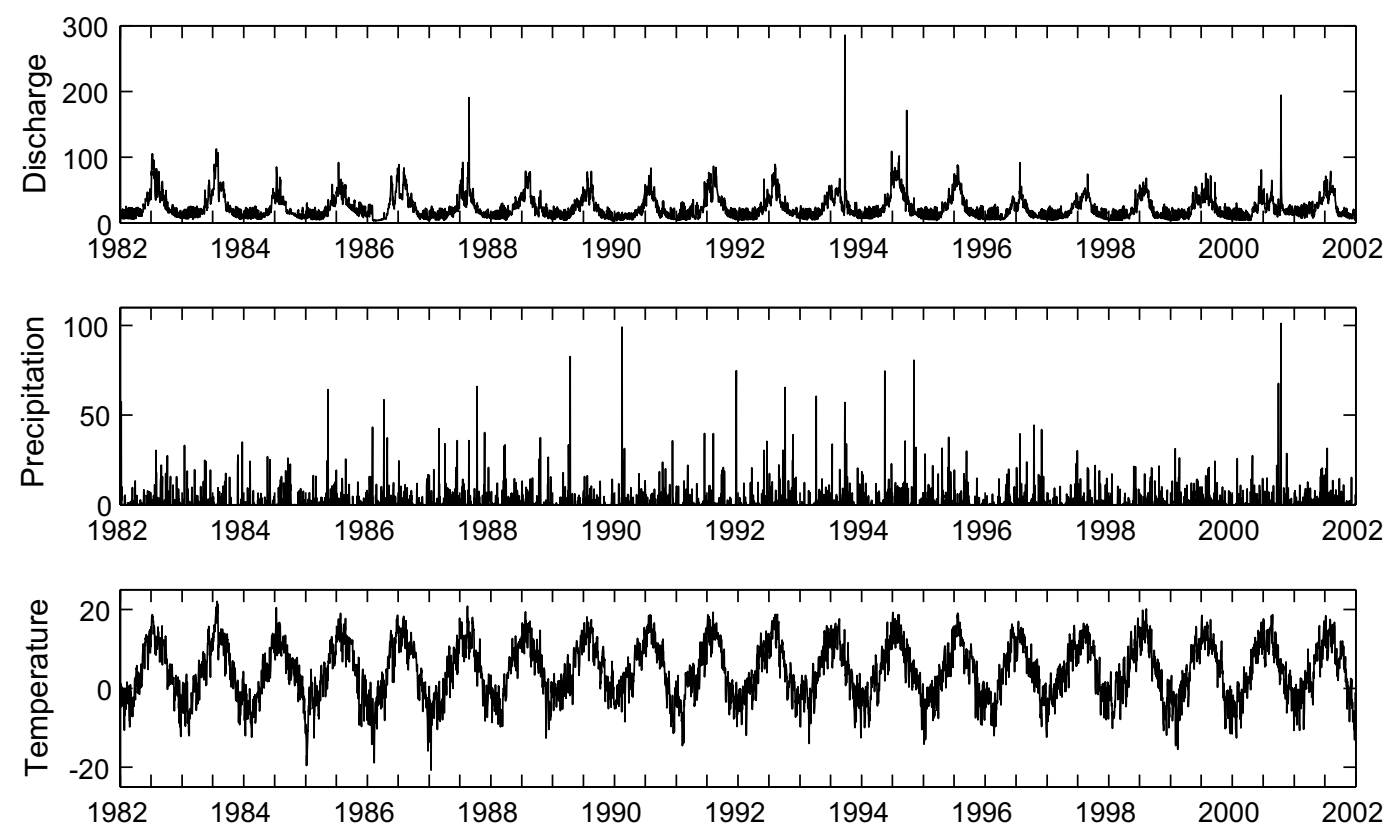

Fig. 2. Observed daily time series of discharge $\left(\mathrm{m}^{3} / \mathrm{s}\right)$, precipitation $(\mathrm{mm} / \mathrm{d})$ and temperature $\left({ }^{\circ} \mathrm{C}\right)$.

catchment at $1638 \mathrm{~m}$ a.s.1. All the observed time series (see Fig. 2) have been pre-treated before the wavelet analysis. They have been deseasonalized, i.e. every date has been normalized by subtracting the interannual mean value of this date and by dividing by the interannual variance. The discharge time series shows a weekly cycle due to the presence of a hydropower plant in the catchment. This weekly cycle has been approximately removed by estimating and subtracting the average weekly cycle and by normalizing to the weekly variance.

As we are interested in correlations between the hydrometeorological processes and not just in coincidences of extreme events (see Section 3.4.7), we have, in addition, transformed the discharge and the precipitation time series to approximately follow a Gaussian marginal: we applied a log-transformation to the discharge series, which - as for many observed river flow series - leads to a good Gaussianity of the data. For the precipitation series, we applied the cubic root, which reduces the skewness of the precipitation distribution and weakens its heavy tail e.g. [33].
The wavelet and wavelet coherence analysis requires defining appropriate Null hypotheses for significance testing. Following Hasselmann [23], we use an AR [1] process for the meteorological times series. The choice of a relevant Null hypothesis for discharge time series is not straightforward as discharge can have rather different autocorrelation structures at different places; many observed discharge series can however be reasonably approximated by an AR [1] process e.g. [34] and we, therefore, chose an AR [1] for all analyzed time series.

\section{Results}

\subsection{Detecting potentially flood producing situations}

For illustrative purposes, Fig. 3 shows the Morlet wavelet spectrum of the observed daily precipitation including the identified areawise significant patches. As for all spectra shown hereafter, this figure also shows the cone of influence above which the results are considerably influenced
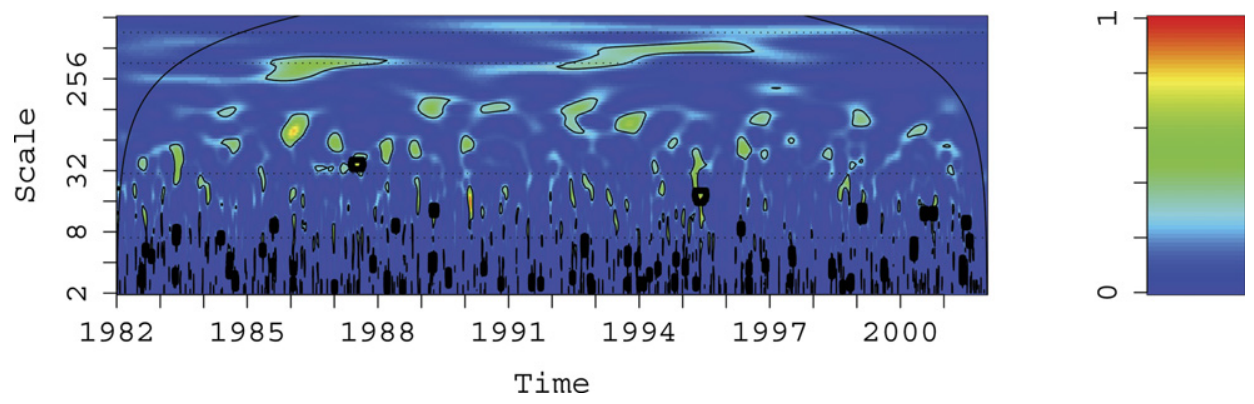

Fig. 3. Wavelet spectrum of daily precipitation; values above the cone of influence (regular black line) are influenced by edge effects; the irregular thin black contour lines delineate pointwise significance patches, the thick black lines delineate areawise significance areas. 
by edge effects (that equals, following Torrence and Compo $[9], \sqrt{2} a$ ). It can easily been seen that this - for this region typical - outcome of continuous wavelet transform of a daily meteorological time series does not provide any easily interpretable insights into the observed process. The numerous low-scale peaks in Fig. 3 probably occur due to the non-Gaussianity discussed in Section 3.3.2.

In this Alpine area, high discharge events usually occur during meteorological situations where warm and humid air masses cross the Alps coming from the South and lead to intense and long precipitation events $[32,35]$. Such situations occur during spring, late summer and autumn but important floods usually only occur in autumn (see Fig. 2) when the catchments are highly saturated and the snow cover that could store part of the rainfall is still small in depth and extent (see, e.g. [27,36]).

These typical meteorological situations are visible in the synoptic pressure fields but are not readily visible in the observed time series of an individual valley such as the Vispa catchment. An analysis of the coherence between the observed precipitation and the temperature time series (Fig. 4a), however, could help detecting these typical mete- orological situations: as expected, patches of high coherence between precipitation and temperature can be seen from spring to autumn, at scales between one and a few days. This example also illustrates that a classical pointwise significance test detects many patches which are small compared to the critical area (i.e. reproducing kernel) and thus likely to be spurious.

It is noteworthy that there is a significant coherence between temperature and precipitation during two major floods $(1993,1994)$ that are known to be the typical outcome of such meteorological situations (see also zoom on 1993-1994 in Fig. 5b). This gives us further confidence that we can detect such southerly flow situations in the wavelet coherence between precipitation and temperature.

As the initial saturation of the catchment plays an important role, it is well known that not all exceptional meteorological situations that bring intense rainfall events during autumn lead to flood situations. As expected based on this considerations, not all significant patches in the coherence between temperature and precipitation in Fig. 4a correspond to high flow situations (Fig. 2). These results show that wavelet coherence analysis of temperature
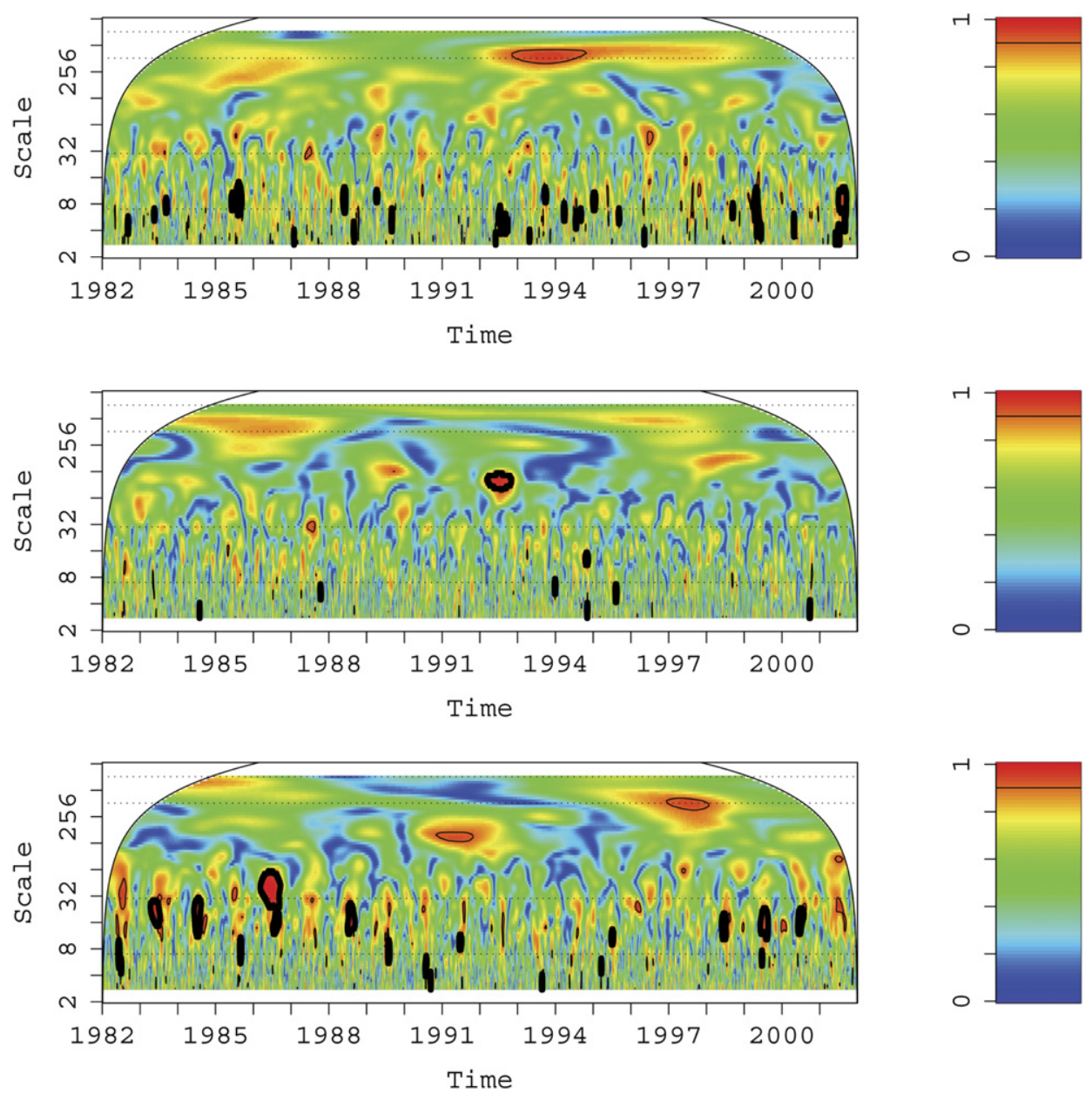

Fig. 4. Squared wavelet sample coherence calculated with a Morlet wavelet, $\omega_{0}=6$, including the pointwise significance test (thin contour line) and the areawise significance test (thick contour line); top: precipitation versus temperature, center: precipitation versus discharge, bottom: temperature vs. discharge of the Vispa River. 

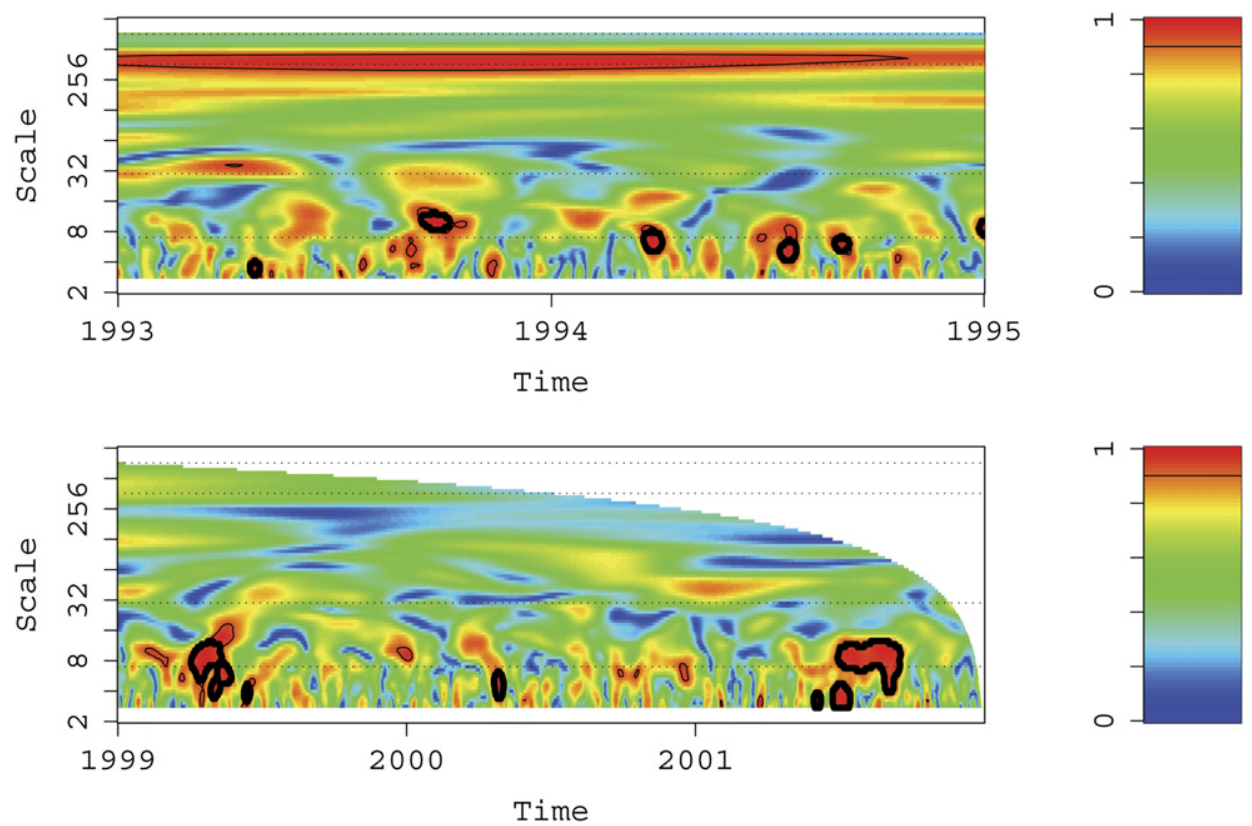

Fig. 5. Squared wavelet sample coherence calculated with a Morlet wavelet, $\omega_{0}=6$ : same as Fig. 4a, but zoom on the years $1993-1994$ (top) and on the year 1999-2001 (bottom).

and precipitation is very useful to help detecting potentially flood triggering situations.

\subsection{Investigating flood producing mechanisms}

The significant coherence between temperature and precipitation during the floods in autumn 1993 and 1994 are in accordance with our (literature-based) knowledge that these floods where the result of exceptional meteorological situations. There are two flood events where we cannot see patches of significant coherence between temperature and precipitation, the one in 1987 and the one in 2000 (see Fig. 4a, Fig. 5a, Fig. 5b). Just as the presence of significant patches does not necessarily imply a meteorological correlation, the absence of patches can only be a hint that the meteorological situation might not have been exceptional.

For the event 2000, where $160 \mathrm{~mm}$ of precipitation where measured during 3 days but the flood was relatively small compared to 1993 (109 mm during 3 days), the snowfall line in this particular catchment is known to haven fallen suddenly during the precipitation event [32]. This explains why for this catchment the flood was small compared to the occurred precipitation.

The coherence between the discharge and the precipitation could be supposed to give us further insights into the dominant processes during all four flood events. Fig. $4 \mathrm{~b}$ shows only very few significant coherence patches between precipitation and discharge. This is to be expected for this catchment: the influence of precipitation on discharge is smoothed out through the snow accumulation (i.e. storage) processes. However, we could still expect significant coherence at very small scales (a few days). Remember, however, that, as discussed in Section 3.3.2, single events are generally not visible in the wavelet spectrum; in this catchment, discharge variations induced by intense precipitation essentially result in such single peaks (Fig. 2). This can be assumed based on process knowledge and visual inspection of the observed times series; it is also confirmed by the wavelet analysis.

Considering the wavelet coherence between discharge and temperature (Fig. 4c), we see numerous significant patches at scales between a few days and a month; the catchment does not seem to significantly react to short temporal fluctuations of temperature. This is a hint that high flow events at the daily scale are not triggered by temperature exclusively, i.e. they are not triggered by snow or ice melt. This interesting conclusion can, contrary to the previous ones, also be obtained using only hydrological process knowledge: in high mountainous and steep catchments (as the one analyzed here), short-term temperature fluctuations only affect small parts of the catchment. If the snowmelt or snowfall line rises momentarily, only very small areas start contributing to the discharge (compared to lowland flat catchments, where a short temperature variation affects large areas simultaneously). Therefore, only temperature variations at higher temporal scales are visible in the discharge time series.

For the event 1987, there are no significant patches in any of the coherence spectra; in addition, only $50 \mathrm{~mm}$ of precipitation were measured during the three preceding days. This suggests that either wavelet spectral analysis is not sensitive enough to detect this event, or this event could have been triggered by circumstances not detectable with the used methods, which could be a hint that the catchment moisture conditions played an important role. 


\section{Discussion}

Estimated wavelet spectra of observed time series can give valuable information about the analyzed processes and their time and scale resolved correlation but their interpretation should be based on appropriate significance tests. The areawise significance test for wavelet spectra developed by Maraun et al. [19] is, therefore, a central point of the presented methods. As they showed, in comparison to the conventional pointwise test, the areawise significance test is slightly less sensitive but more specific, i.e. the probability of obtaining misleading false positive results has been reduced dramatically.

For the hydrological case study presented here, this characteristic of the areawise test enables interpretations of the estimated spectra that can be related in a meaningful way to physical process knowledge. Applied to the coherence between precipitation and temperature, it contributes to identify potentially flood producing meteorological situations not readily visible in the original data, and to generate hypotheses about flood triggers (to test these hypotheses much more detailed investigations are, of course, required). How important the use of such an areawise test is, can best been seen in the coherence between temperature and precipitation (Fig. 4a), where a pointwise test would identify numerous small significant patches that have no hydrological meaning.

This shows that wavelet spectral analysis, combined to sound significance testing, is a powerful tool to assist the identification of dominant hydrological processes in view of process understanding and model development. For the present case study, the identified potentially flood producing meteorological situations could now be used to focus the model development and its performance assessment on the reproduction of the discharge during these critical situations (around 20 situations, see Fig. 4a). This would in particular reveal whether the hydrological model is able to explain the occurrence and the non-occurrence of floods during potentially flood producing situations, which would give detailed insights into the model performance. This represents a major advancement compared to simply assess the model performance over the entire observed period or only for the observed four flood events.

In addition, the performance of the weather generator used for the design flood estimation should now be reassessed to test whether the coherence of the simulated temperature and precipitation time series differ significantly from the observed pattern of coherence. This opens very new perspectives for the performance assessment of weather generators (see, e.g. $[29,37])$.

\subsection{Some remarks on the interpretation of wavelet spectra}

As mentioned earlier (see Section 3.3.2), local strong non-Gaussianity of the data can lead to patches in the coherence spectrum that are not related to a particular correlation structure but to a coincidence of extreme events. In addition to the transformations mentioned in Section 4, we tested the effect of rendering the precipitation series almost perfectly Gaussian by applying the normal quantile transformation [38]. This completely suppresses the heavy tail of the distribution and, as a result, removes most of the significant patches in Fig. 3 as well as, for instance, the significant patches in 1994 in Fig. 5a, i.e. in the latter case, the patches probably correspond to a coincidence of extreme events rather than a co-oscillation. Some of the patches might, however, disappear due to effects of the transformation on the local correlation structure: De Oliveira [39] has shown that transforming a stationary random field to a Gaussian marginal distribution effects the autocorrelation function only marginally. To our knowledge, it has, however, not been studied how strong this effect might be locally, especially in the presence of heavy tails.

As any statistical test, the areawise test also produces false positive results. To increase confidence in the obtained results, the total area of the areawise significant results should be compared to the total area of the pointwise significant results. If there is a large area of pointwise patches but only a small area of areawise ones, the latter might well be false positive outliers.

The areawise test can also be simply too specific, but corresponding cases are very easily identified by eye: assume a true deviation from the Null hypothesis over a certain length that is large compared to the critical area. The noisy wavelet periodogram might "frazzle" a corresponding patch and accordingly, the pointwise test might split this single deviation into two separate patches, which are both small compared to the critical area. As a result, no critical area fits in and the areawise test produces a false negative result. Looking closely at neighboring pointwise significant patches that might add up to an areawise significant patch can help detecting such situations.

\section{Conclusion}

For the presented case study from the Swiss Alps, wavelet spectral analysis of precipitation, temperature and discharge offers a powerful tool to help detecting potentially flood producing meteorological situations and to distinguish between different types of floods with respect to the prevailing critical hydrometeorological conditions. However, the obtained results and drawn conclusions are only possible based on a sound combination of previous knowledge about the physical processes involved and a methodologically rigorous wavelet analysis framework.

In fact, the application and interpretation of these relatively new mathematical methods is not as straightforward as suggested by some case studies. Therefore, this paper presents an overview of state-of-the-art methods and recent developments in wavelet spectral analysis before applying them to the hydrological case study. 
In summary, the key points to which should be paid attention for any application of wavelet spectral analysis to hydrological time series are the following:

- Continuous wavelet analysis transforms a one dimensional time series to the two dimensional time-scale domain. This results in inherent correlations in the spectra given by the reproducing kernel of the chosen wavelet. These correlations reflect the fact that time and frequency cannot be jointly estimated with arbitrary precision but are subject to an uncertainty relation. Therefore, the visual interpretation of estimated spectra can be misleading and significance testing is not straightforward.

- The interpretation of estimated wavelet spectra requires confidence intervals or formal significance testing that should be based on appropriate statistical tests, such as the areawise significance test of Maraun et al. [19] presented here, and on reasonable Null hypotheses.

- Cross spectra are non-normalized measures of the time and scale resolved covariance; therefore, they are only interpretable in conjunction with appropriate confidence intervals. We recommend to calculate the wavelet coherence instead.

- Wavelet spectral analysis enables the inference of time and scale resolved correlations between two time series through the estimation of wavelet coherences; as for the sample spectra, their significance should be tested.

- A significant coherence in a short time interval can be due to spurious common oscillations; i.e. a short coherence interval alone is not necessarily indicative of a physical relationship.

Wavelet spectral analysis has the potential to substantially contribute to the understanding and modelling of hydrological behavior. The results presented here for the time and scaled resolved analysis of hydrometeorological time series from the Swiss Alps are merely qualitative; the obtained insights will however be of substantial help for the improvement of simulation tools for flood prediction in this area and they will namely build the basis for the development of new model performance diagnosis tools.

We believe that given the state-of-the-art of wavelet spectral analysis, the time is ripe for a shift from merely qualitative to such more quantitative hydrological applications, for example for model diagnostics but also for the development of signatures of hydrological behavior (see, e.g. [40]) or of new catchment classification methods (see, e.g. [41]).

Such more quantitative applications of wavelet spectral analysis require however further research into the interpretation of continuous wavelet spectra for hydrological processes and should be completed in close collaboration with time series analysts from other fields.

All methods presented here are implemented in SOWAS (software for wavelet spectral analysis and synthesis) writ- ten in R and Matlab and freely available on http://tocsy. agnld.uni-potsdam.de/wavelets.

\section{Acknowledgements}

The research of two authors was supported by a research fellowship: the first author was supported by the Swiss National Science Foundation SNF, the second author by the German Science Foundation DFG, SFB 555 . We also wish to thank the Swiss national weather service, MeteoSwiss, for providing the meteorological time series and the Swiss Federal Office for the Environment (Hydrological Section) for providing the discharge time series. Many thanks to Jonas Laehnemann for translating the sowas software from $\mathrm{R}$ to Matlab. The quality of this paper has been greatly improved by the constructive comments of four anonymous reviewers.

\section{References}

[1] Favre AC, El Adlouni S, Perreault L, Thiémonge N, Bobé B. Multivariate hydrological frequency analysis using copulas. Water Resour Res 2004;40:W01101. doi:10.1029/2003WR002456.

[2] Grossmann A, Morlet J. Decomposition of Hardy functions into square integrable wavelet constant shape. SIAM J Math Anal 1984;15(4):723-36

[3] Gabor D. Theory of communication. J IEEE 1946;93:429-57.

[4] Daubechies I. Ten lectures on wavelets. Soc Indust Appl Math 1992.

[5] Holschneider M. Wavelets: an analysis tool. Oxford University Press; 1995.

[6] Mallat S. A wavelet tour of signal processing. Academic Press; 1999.

[7] Kumar P, Foufoula-Georgiou E. Wavelet analysis for geophysical applications. Rev Geophys 1997;35(4):385-412.

[8] Foufoula-Georgiou E, Kumar P, editorsWavelets in geophysics. San Diego: Academic Press; 1994.

[9] Torrence C, Compo GP. A practical guide to wavelet analysis. Bull Am Meteorolog Soc 1998;79(1):61-78.

[10] Coulibaly P, Burn DH. Wavelet analysis of variability in annua Canadian streamflows. Water Resour Res 2004;40(3):W03105. doi:10.1029/2003WR002667.

[11] Labat D, Ronchail J, Guyot JL. Recent advances in wavelet analyses: Part 2 - Amazon, Parana, Orinoco and Congo discharges time scale variability. J Hydrol 2005;314(1-4):289-311.

[12] Zhang Q, Xu Ch, Jiang T, Wu Y. Possible influence of ENSO on annual maximum streamflow of the Yangtze River. Chin J Hydrol 2007;333(2-4):265-74. doi:10.1016/j.jhydrol.2006.08.010.

[13] Gaucherel C. Use of wavelet transform for temporal characterisation of remote watersheds. J Hydrol 2002;269(3-4):101-21.

[14] Lafreniere M, Sharp M. Wavelet analysis of inter-annual variability in the runoff regimes of glacial and nival stream catchments, Bow Lake, Alberta. Hydrol Proc 2003;17:1093-118.

[15] Labat D, Ababou R, Mangin A. Rainfall-runoff relations for karstic springs. Part II: continuous wavelet and discrete orthogonal multiresolutions analyses. J Hydrol 2000;238(3-4):149-78.

[16] Smith MB, Koren VI, Zhang Z, Reed SM, Pan J-J, Moreda F. Runoff response to spatial variability in precipitation: an analysis of observed data. J Hydrol 2004;298(1-4):267-86.

[17] Si BC, Zeleke TB. Wavelet coherency analysis to relate saturated hydraulic properties to soil physical properties. Water Resour Res 2005;41(11):W11424. doi:10.1029/2005WR004118.

[18] Lane SN. Assessment of rainfall-runoff models based upon wavelet analysis. Hydrol Proc 2006;21:586-607. doi:10.1002/hyp.6249.

[19] Maraun D, Kurths J, Holschneider M. Non-stationary gaussian processes in wavelet domain: definitions estimation and significance testing. Phys Rev Lett 2007;75(1):PRE 028701. 
[20] Maraun D, Kurths J. Cross wavelet analysis: significance testing and pitfalls, Nonlinear Processes in Geophysics. Nonlin Proc Geophys 2004;11(4):505-14.

[21] Carmona R, Hwang W-L, Torresani B. Practical time-frequency analysis gabor and wavelet transforms with an implementation in S. Academic Press; 1998.

[22] Davison AC, Hinkley DV. Bootstrap methods and their application. Cambridge series in statistical and probabilistic mathematics. Cambridge University Press; 1997.

[23] Hasselmann K. Stochastic climate models: part I. theory. Tellus 1976;28(6):473-85.

[24] Huang JP, Higuchi K, Shabbar A. The relationship between the North Atlantic oscillation and El Nino Southern oscillation. Geophys Res Lett 1998;25(14):2707-10.

[25] Jordan F. Modèle de prévision et de gestion des crues - Optimisation des opérations des aménagements hydroélectriques accumulation pour la réduction des débits de crue. Ph.D thesis, Ecole Polytechnique Fédérale de Lausanne, Switzerland; 2006.

[26] Schaefli B, Hingray B. Mezghani A, Hamdi Y, Guex F, Niggli M, Musy A. Estimation des débits de crue du Rhône jusqu'a Porte-duScex - Rapport de synthèse réalisé dans le cadre du projet CONSECRU 2. Technical report, Ecole Polytechnique Fédérale de Lausanne, Switzerland; 2006.

[27] Schaefli B, Hingray B, Niggli M, Musy A. A conceptual glaciohydrological model for high mountainous catchments. Hydrol Earth Syst Sci 2005;9:95-109.

[28] Mezghani B, Hingray A. Musy. Modèle de Génération spatiotemporelle des variables météorologiques journalières et horaires - Rapport de synthèse réalisé dans le cadre du projet CONSECRU 2. Technical report, Ecole Polytechnique Fédérale de Lausanne, Switzerland, 2006.

[29] Beersma JJ, Buishand TA. Multi-site simulation of daily precipitation and temperature conditional on the atmospheric circulation. Climate Res 2003;25(2):121-33.
[30] Obled C, Bontron G, Garcon R. Quantitative precipitation forecasts: a statistical adaptation of model outputs through an analogues sorting approach. Atmos Res 2002;63(3-4):303-24.

[31] Stoffel M, Lièvre I, Conus D, Grichting MA, Raetzo H, Gärtner HW, et al. 400 Years of debris-flow activity and triggering weather conditions: Ritigraben, Valais, Switzerland. Arctic Antarc Alpine Res 2005;37(3):387-95.

[32] Swiss Federal Office for Water and Geology, Hochwasser $2000-$ Ereignisanalyse/Fallbeispiele, Berichte des BWG, Serie Wasser, Nr. 2, Bern, Technical report, 2002.

[33] Katz RW. Extreme value theory for precipitation: sensitivity analysis for climate change. Adv Water Resour 1999;23:133-9.

[34] A. Montanari, E. Toth, Calibration of hydrological models in the spectral domain: an opportunity for ungauged basins? Water Resour Res 43: W05434. doi:10.1029/2006WR005184.

[35] Frei C, Schär C. A precipitation climatology of the Alps from highresolution rain-gauge observations. Int J Climatol 1998;18:873900.

[36] Frei C, Gurtz J, Schär C. Climate dynamics and extreme precipitation and flood events in Central Europe. Integr Assess 2000;1:281-99.

[37] Buishand TA, Brandsma T. Multisite simulation of daily precipitation and temperature in the Rhine basin by nearest-neighbor resampling. Water Resour Res 2001;37(11):2761-76.

[38] Kelly KS, Krzysztofowicz R. A bivariate meta-Gaussian density for use in hydrology. Stochas Hydrol Hydraul 1997;11:17-31.

[39] De Oliveira V. A note on the correlation structure of transformed Gaussian random fields. Aust NZ J Stat 2003;45(3):353-66.

[40] Sivapalan M. Encyclopedia of Hydrological Sciences. Chapter Pattern process and function: elements of a unified theory of hydrology at the catchment scale. Chichester: Wiley; 2005. p. 193220.

[41] McDonnell JJ, Woods R. On the need for catchment classification. J Hydrol 2004;299:2-3. 